\title{
A NOTE ON COUNTING ISOTROPY SUBGROUPS
}

\author{
L. N. MANN
}

1. Introduction. An elementary p-group of rank $k$ is a group isomorphic to the direct sum of $k$ copies of $Z_{p}$, the additive group of integers modulo a prime $p$. P. A. Smith has investigated the actions of such a group $G$ of homeomorphisms on the $n$-sphere $S^{n}$ and has observed certain similarities with the standard orthogonal actions. In particular, he has shown [4], [5] that if $G$ acts effectively on $S^{n}$, then $k=\operatorname{rank} G \leqq[(n+1) / 2]$ for $p$ odd and $k \leqq n+1$ for $p=2$. As usual, $[x]$ denotes the largest integer not exceeding $x$. The purpose of this note is to show that if such a group $G$ acts effectively on $S^{n}$, then the number of distinct isotropy subgroups cannot exceed $2^{[(n+1) / 2]}$ for $p$ odd, and $2^{n+1}-1$ for $p=2$. These are precisely the bounds which exist for orthogonal actions.

The proof proceeds by first observing that every maximal isotropy subgroup is of rank $k-1$. A formula of Borel [1] is then utilized to show that the number of maximal isotropy subgroups cannot exceed $[(n+1) / 2]$ for $p$ odd, $n+1$ for $p=2$, and that, moreover, each isotropy subgroup of rank $k-i, 1 \leqq i \leqq k-1$, is the intersection of $i$ maximal isotropy subgroups. Noting that $k \leqq[(n+1) / 2]$ for $p$ odd and $k \leqq n+1$ for $p=2$, and allowing for the isotropy subgroups $G$ and $\{e\}$, the result follows.

2. Definitions and preliminaries. Given an action of a topological transformation group $G$ on a space $X$, the isotropy subgroup at a point $x_{0} \in X$, denoted by $G_{x_{0}}$, is defined as the subgroup of all elements of $G$ which leave $x_{0}$ fixed. The action is said to be effective if $\bigcap_{x \in X} G_{x}=e$, the identity element of $G$; it is said to be free if $\{e\}$ is the only iso. tropy subgroup. The fixed-point set of the action, denoted by $F(G, X)$, is the subset of $X$ of all points with isotropy subgroup $G$.

All spaces considered will be compact Hausdorff spaces and the usual Čech cohomology will be used. A cohomology n-sphere over $Z_{p}$ is a space with the cohomology groups, coefficient group $Z_{p}$, of $S^{n}$. A generalized cohomology $n$-sphere over $Z_{p}$ is a cohomology $n$-sphere over $Z_{p}$ which is also a cohomology $n$-manifold over $Z_{p}[1]$. Results of Smith [2], [3], [5] show that if an elementary p-group $G$ acts on a cohomology $n$-sphere (generalized cohomology $n$-sphere) $X$ over $Z_{p}$, then $F(G, X)$ is a cohomology $r$-sphere (generalized cohomology 1964.

Presented to the Society, January 23, 1964; received by the editors March 10, 
$r$-sphere) over $Z_{p}, r \leqq n$. Moreover, if $X$ is a generalized cohomology $n$-sphere and the action is effective, then $r \leqq n-2$ and $n-r$ is even for $p$ odd, and $r \leqq n-1$ for $p=2$. We shall use strongly the following result of Borel [1]. (For $F(K, X)$ empty, it is agreed that $n(K, X)$ $=-1$.)

TheOREM 1 (BOREL). Suppose $G$ is an elementary p-group acting effectively on a cohomology $n$-sphere $X$ over $Z_{p}$. For each subgroup $K$ of $G$ let $n(K, X)$ be the integer such that $F(K, X)$ is a cohomology $n(K, X)$ sphere over $Z_{p}$. Then

$$
n-n(G, X)=\sum_{H}(n(H, X)-n(G, X)),
$$

where $H$ runs through the subgroups of index $p$ in $G$.

It will prove useful to have available the following group-theoretic result which we present without proof.

LEMMA 1. Let $G$ be an elementary p-group of rank $k$ and $K$ a subgroup of $G$ of rank $k-i, 1 \leqq i \leqq k-1$. Suppose $K$ is the intersection of $t$ distinct subgroups of $G$, each of rank $k-1$. Then $t \geqq i$ and $K$ is the intersection of some subcollection of $i$ of the $t$ subgroups.

\section{Main results.}

TheOREM 2. Suppose $G$ is an elementary p-group acting effectively on a generalized cohomology $n$-sphere $X$ over $Z_{p}$. Let $r=n(G, X) \geqq-1$. Then the number of distinct isotropy subgroups cannot exceed $2^{(n-r) / 2}$ for $p$ odd or $2^{n-r}$ for $p=2$.

Proof. Suppose rank $G=\Gamma(G)=k$. We shall say that an isotropy subgroup of rank less than $k$ is maximal if it is not properly contained in any isotropy subgroup with the possible exception of $G$. We first show that each maximal isotropy subgroup is of rank $k-1$. Suppose, on the contrary, that $S$ is a maximal isotropy subgroup with $\Gamma(S)$ $\leqq k-2$. Now $G / S$ leaves the generalized cohomology sphere $F(S, X)$ invariant. Moreover, since $S$ is a maximal isotropy subgroup, it is easy to see that $G / S$ acts freely outside of the fixed-point set on $F(S, X)$. In fact, $F(G / S, F(S, X))=F(G, X)$. Now $F(S, X) \supset F(G, X)$ $=F(G / S, F(S, X))$ and the action of $G / S$ on $F(S, X)$ is effective. We apply Theorem 1 to this action since $\Gamma(G / S) \geqq 2$. We have

$$
n(S, X)-n(G, X)=\sum_{H}(n(H, F(S, X))-n(G, X)),
$$

where $H$ runs through the subgroups of index $p$ in $G / S$. Recalling that $G / S$ acts freely outside of $F(G, X)$ on $F(S, X)$, it follows that 
$F(H, F(S, X))=F(G, X)$ for all $H$ and, therefore, the right-hand side of (1) is zero. However, since $G / S$ acts effectively on $F(S, X)$, it follows that the left-hand side of (1) is strictly positive, giving us a contradiction. Hence, each maximal isotropy subgroup is of rank $k-1$. This conclusion could also have been obtained by using the results of $[4]$.

Next we observe that there are at most $(n-r) / 2$ maximal isotropy subgroups for $p$ odd. But this follows immediately from Theorem 1 since each term $(n(H, X)-n(G, X))$ must be even for an odd prime. For $p=2$, we conclude that there are at most $n-r$ maximal isotropy subgroups.

Suppose that $T \subset S \subset G, \Gamma(S)=k-1$, and $\Gamma(T)=k-2$, with $S$ an isotropy subgroup of the action of $G$ on $X$ and $T$ an isotropy subgroup of the action of $S$ on $X$. We wish to conclude that $T$ is also an isotropy subgroup of the action of $G$ on $X$. We know that $F(T, X)$ $\supset F(S, X)$. Suppose $T$ is not an isotropy subgroup of $G$ on $X$. Then

$$
F(T, X) \subseteq \bigcup_{i} F\left(S_{i}, X\right),
$$

for some collection of isotropy subgroups $S_{i}$ of $G$ on $X$ where $S_{i} \supset T$ and $\Gamma\left(S_{i}\right)=k-1$ for each $i$. Since $S_{i} \supset T$, we have $F(T, X) \supseteq F\left(S_{i}, X\right)$ and

$$
F(T, X)=\bigcup_{i} F\left(S_{i}, X\right)
$$

Due to dimensional restrictions (we are dealing with connected cohomology manifolds), we must have $F(T, X)=F\left(S_{i_{0}}, X\right)$ for some $i_{0}$. Hence, $F\left(S_{i_{0}}, X\right)=F(T, X) \supset F(S, X)$ which contradicts $S$ being an isotropy subgroup.

We now come to the crux of the argument: to show that if $R$ is an isotropy subgroup of $G$ of rank $k-i, 1 \leqq i \leqq k-1$, then $R$ is the intersection of $i$ distinct isotropy subgroups of rank $k-1$. By Lemma 1 , it is sufficient to show that $R$ is the intersection of some collection of isotropy subgroups of rank $k-1$. We consider first the case that $\Gamma(k)$ $=k-2$. It is sufficient to exhibit two distinct maximal isotropy subgroups which contain $R$. Consider the action of $G / R, \Gamma(G / R)=2$, on the generalized cohomology sphere $F(R, X)$. We have $F(G / R, F(R, X))=F(G, X) \subset F(R, X)$. As $R$ is an isotropy subgroup, this action must be effective. Applying Theorem 1 to the action, one sees that there must exist distinct cyclic subgroups $K_{1}^{*}$ and $K_{2}^{*}$ of $G / R$ with $F\left(K_{j}^{*}, F(R, X)\right) \supset F(G, X)$ for $j=1$, 2. Since $K_{1}^{*}$ and $K_{2}^{*}$ generate $G / R, F\left(K_{1}^{*}, F(R, X)\right) \neq F\left(K_{2}^{*}, F(R, X)\right)$; for, 
otherwise, $F(G / R, F(R, X))$ would be $F\left(K_{1}^{*}, F(R, X)\right)$ instead of $F(G, X)$. Let $\pi$ be the projection $\pi: G \rightarrow G / R$, and let $S_{j}=\pi^{-1}\left(K_{j}^{*}\right)$, $j=1,2$. We claim $F\left(S_{1}, X\right) \neq F\left(S_{2}, X\right)$ and $F\left(S_{j}, X\right) \supset F(G, X)$ for $j=1,2$. To see this observe that $F\left(S_{j}, X\right)=F\left(S_{j} / R, F(R, X)\right)$ $=F\left(K_{j}^{*}, F(R, X)\right)$. Now suppose that $R=G_{x_{0}}$. Then there exists $y_{j} \in F\left(S_{j}, X\right)$ with $y_{j} \neq x_{0}$ and $y_{j} \notin F(G, X), j=1,2$; and, moreover, $y_{1} \neq y_{2}$. It follows that $S_{j}=G_{y_{j}}, j=1,2$, and we have two distinct maximal isotropy subgroups, $S_{1}$ and $S_{2}$, containing $R$.

We proceed by induction on $k=\Gamma(G)$, starting with $k=3$. But if $\Gamma(G)=3$, we need consider isotropy subgroups $R$ only of rank $k-2$ (that is, of rank 1), and the argument above immediately applies. Suppose then that $R$ is an isotropy subgroup of $G, \Gamma(G)=k$. Then there exists a maximal isotropy subgroup $S$ of $G$ with $R \subset S$ and $\Gamma(S)=k-1$. Consider the action of $S$ on $X$. By our induction hypothesis, $R$ is the intersection of a collection $T_{j}$ of isotropy subgroups of $S$ of rank $k-2$. By an argument above, each $T_{j}$ is also an isotropy subgroup of the action of $G$ on $X$ and, therefore, the intersection of two maximal isotropy subgroups of rank $k-1$. Finally, then, $R$ is the intersection of a collection of isotropy subgroups, each of rank $k-1$.

We now know that there are at most binomial coefficient $\left(\begin{array}{c}(n-r) / 2 \\ i\end{array}\right)$ distinct isotropy subgroups of rank $k-i, 1 \leqq i \leqq k-1$, for $p$ odd; at most $\left(\begin{array}{c}n-r \\ i\end{array}\right)$ distinct ones for $p=2$. Noting that $k \leqq(n-r) / 2$ for $p$ odd and $k \leqq n-r$ for $p=2$, an immediate generalization of Smith's result in [4], [5], and allowing for the isotropy subgroups $G$ and $\{e\}$ of rank $k$ and 0 , respectively, the theorem follows. Actually, if $r=-1$, that is, $F(G, X)$ is empty, then we may omit $G$ as a possible isotropy subgroup and we observe that there exist at most $2^{(n+1) / 2}-1$ distinct isotropy subgroups for $p$ odd, and at most $2^{n+1}-1$ for $p=2$.

CoROLlaRy. Let $G$ be an elementary p-group acting effectively on $E^{n}$, euclidean n-space. Then the number of distinct isotropy subgroups cannot exceed $2^{[n / 2]}$ for $p$ odd and $2^{n}$ for $p=2$.

Proof. Extend the action of $G$ to $S^{n}$ by leaving the point at infinity fixed. Of course, a stronger statement of the Corollary is possible in terms of the cohomology dimension, $r$, of $F\left(G, E^{n}\right)$.

\section{REFERENCES}

1. A. Borel et al., Seminar on transformation groups, Princeton Univ. Press, Princeton, N. J., 1960.

2. P. A. Smith, Transformations of finite period, Ann. of Math. (2) 39 (1938), 127-164.

3. - Transformations of finite period. II, Ann. of Math. (2) 40 (1939), 690-711. 
4. - Permutable periodic transformations, Proc. Nat. Acad. Sci. U.S.A. 30 (1944), 105-108.

5. - New results and old problems in finite transformation groups, Bull. Amer. Math. Soc. 66 (1960), 401-415.

INSTITUTE FOR DEFENSE ANALYSES AND

UNIVERSITY OF VIRGINIA

\title{
DIFFERENTIABLE ACTIONS OF COMPACT ABELIAN LIE GROUPS ON $S^{n}$
}

\author{
L. N. MANN
}

1. Introduction. In [9] P. A. Smith raises the following question: If a finite group $G$ acts effectively on the $n$-sphere $S^{n}$, must there also be some effective orthogonal action of $G$ on $S^{n}$ ? Stated another way, must all finite groups acting effectively on $S^{n}$ be isomorphic to subgroups of the orthogonal group $O(n+1)$ ? Smith has answered this question in the affirmative for the case where $G$ is an elementary p-group [8], [9]. The Corollary to Theorem 2 of this paper settles the case where $G$ is a compact abelian Lie group (in particular, a finite abelian group) and the action is assumed differentiable.

The proof of our main result is immediate if one assumes the existence of a fixed point, as evidenced by the following result which utilizes Bochner's theorem on local linearity about a fixed point.

Theorem 1. Let $G$ be a compact Lie group operating effectively and differentiably on a differentiable $n$-manifold $X$. If there exits a point $x_{0}$ left fixed by every element of $G$, then $G$ is isomorphic to a subgroup of $O(n)$.

Proof. By Bochner's theorem [5, p. 206], we may assume $G$ acts orthogonally (but not necessarily effectively) on some small closed $n$ cell $D$ with center $x_{0} . G$ leaves bdy $D=S^{n-1}$ invariant. If $G$ is not effective on $S^{n-1}$, then there must be a homeomorphism $g_{0}$ of finite order in $G$ which leaves $S^{n-1}$ pointwise fixed. Since $g_{0}$ acts linearly on $D$ and leaves $x_{0}$ fixed, it must also leave $D$ pointwise fixed. By Newman's theorem [5, p. 223], $g_{0}$ must leave $X$ pointwise fixed, violating the effectiveness of $G$ on $X$. Hence $G$ acts orthogonally and effectively on $S^{n-1}$, and the theorem follows.

Received by the editors March 10, 1964. 